\title{
Universal modesty in signal-burying games
}

Tadeg Quillien

November 12, 2018

\section{Contents}

$\begin{array}{lr}\text { Abstract } & 2 \\ \text { Introduction } & 2 \\ 1 \text { Model } & 3 \\ 2 \text { Standard burying equilibrium } & 4 \\ 3 \text { Pooling burying equilibrium } & 5 \\ 4 \text { Individual-based simulations } & 7 \\ 5 \text { Analytical model with mistakes } & 9 \\ 6 \text { Discussion } & 10 \\ 7 \text { Conclusion } & 11 \\ \text { References } & 12 \\ \text { Appendix } & 13\end{array}$




\begin{abstract}
Why would people hide positive information about themselves? Evolutionary game theorists have recently developed the signal-burying game as a simple model to shed light on this puzzle; they have shown that the game has an equilibrium where some agents are better off deliberately reducing the visibility of the signal by which they broadcast their positive traits. However, their explanation falls short of explaining all modesty norms, since this equilibrium also features individuals who openly brag. This leaves modesty norms that everyone adheres to in want of an explanation. Here we show that the signal-burying framework actually affords such an explanation: the game contains an equilibrium where all agents who send a signal voluntarily reduce its conspicuousness. Surprisingly, the stability of the two kinds of equilibria rely on very different principles. The equilibrium where some agents brag is stable because of costly signaling dynamics. By contrast, the universal modesty equilibrium exists because buried signals contain probabilistic information about a sender's type, and receivers make optimal use of this information. In the latter equilibrium, burying a signal can be understood as a handicap which makes the signal more honest, but honesty is not achieved through standard costly signaling dynamics.
\end{abstract}

\title{
Introduction
}

In Stanley Kubrick's classic movie Dr Strangelove, the eponymous scientist is surprised to learn that the Soviets did not advertise their deterrence device: "Of course, the whole point of a Doomsday Machine is lost, if you keep it a secret! Why didn't you tell the world?". Behavioral scientists are in a same state of perplexity with respect to many aspects of human behavior. Philanthropists often make massive donations under cover of anonymity, and observers consider such anonymous giving more virtuous [1]. Artists hide some of the meaning of their work as easter eggs for spectators to discover, novelists publish some of their books under pseudonyms, and fashionistas sometimes buy expensive designer clothes that do not feature the brand logo. More generally, people are reluctant to advertise their positive traits too openly. These phenomena

seem to run contrary to standard evolutionary and economic explanations of human behavior, which hold that generosity, artistic pursuits, and conspicuous consumption function in large part to attract and impress cooperation and romantic partners $[2,3,4,5,6,7]$. Since the whole point about prowesses and good deeds is lost if we keep them a secret, why don't we tell the world?

In a recent paper, Hoffman, Hilbe \& Nowak argue that modesty sometimes does make functional sense [8]. They introduce a simple formal model, the signal-burying game, and find equilibria of the game where some individuals are better off reducing the conspicuousness of their broadcasts of positive traits. Their main insight is that the act of deliberately making a signal hard to notice 
is itself a signal: it conveys the message that you are confident that people will find out anyway, or that you do not really care about reaching out to those who would miss the hidden message.

At the equilibrium that Hoffman et al describe, only a fraction of individuals who send a signal reduce its conspicuousness; by necessity, the equilibrium also features individuals who openly advertise their positive traits. Here we show that their game-theoretic framework also has the potential to explain situations where a modesty norm is observed by everyone. We first describe the setting of the signal-burying game. Then we present the original equilibrium found by Hoffman et al, followed by the new pooling equilibrium.

\section{Model}

The signal-burying game pairs a sender with a receiver; the sender tries to convince the receiver to interact with him: if a receiver agrees to interact with the sender, the latter receives a payoff of 1 . The receiver, however, only wants to interact with certain types of senders, but a sender's type is only known to the sender. Senders can be of High, Medium, or Low quality. Likewise, receivers vary in their selectivity. Strong receivers only want to interact with High senders: they get a payoff of 1 when interacting with a High sender, and a payoff of -1 when interacting with a Medium or Low sender. Weak receivers are less selective: they get a payoff of 1 when interacting with a High or Medium sender, and -1 with a Low sender. If the receiver declines to interact, both players get a null payoff. The sender is a High, Medium, or Low type with probability $p_{h}$, $p_{m}$, or $p_{l}$, respectively. The receiver is Strong or Weak with probability $q_{s}$ or $q_{w}$. These probability distributions are common knowledge, but a player's type is only known to that player. ${ }^{1}$

Senders can send a signal, if they wish. They have three options: send a buried signal, a clear signal, or remain silent. By assumption, signaling is prohibitively costly for Low types, but is free for High and Medium types. Therefore, by sending a signal, a High or Medium type can broadcast positive information about himself (namely, that he is not a Low type). Senders also have the option of making their signal hard to detect: if they choose to bury their signal, it is only revealed to the receiver with probability $r_{h}$ (if it was sent by a High sender) or $r_{m}$ (if it was sent by a Medium sender). If it is detected, the receiver knows that the signal was buried.

The interesting feature of the game resides in the fact that both High and Medium types are able to send a signal, and therefore a receiver cannot distinguish between these two types if she receives a clear signal. We thus make

\footnotetext{
${ }^{1}$ For simplicity of exposition, we omit some of the parameters used in the original model by Hoffman et al.
} 
the final assumption that Medium senders outnumber High senders in the population $\left(p_{h}<p_{m}\right)$, such that when both types send clear signals, a Strong receiver would get a negative expected payoff (namely $p_{h}-p_{m}$ ) for interacting with a player sending a clear signal. The main insight of Hoffman et al. is that High types can distinguish themselves from Medium types, and thereby attract Strong receivers, by making their signal harder to detect. We describe their result in the next section; in the following section we show the existence of another kind of burying equilibrium.

\section{Standard burying equilibrium}

Under certain conditions, there exists an equilibrium where High senders have incentives to send buried signals, while medium senders are better off sending clear signals. As a result, only High senders send buried signals; therefore burying reliably conveys sender quality. Then, Strong receivers only accept buried signals, while Weak receivers accept both kinds of signals (nobody accepts silent senders). The equilibrium exists when:

$$
\begin{aligned}
& r_{h}>q_{w} \\
& r_{m}<q_{w}
\end{aligned}
$$

The first condition says that High senders are better off sending a buried signal. Their buried signal is detected with probability $r_{h}$, and when this happens the receiver accepts the interaction. Their clear signal is always detected, but only Weak receivers (who make up a proportion $q_{w}$ of the population) accept the interaction. By a similar logic, the second condition says that Medium senders are better off sending a clear signal. Note that (1)(2) imply that $r_{h}>r_{m}$ : buried signals from High types are more likely to be detected than those from Medium types. This could represent, for instance, the fact that among people who make charitable donations, some are well-connected enough that they could more easily arrange for their identity to leak if they chose to donate anonymously.

A Strong receiver who deviates from the strategy by accepting clear signals is worse off, because only Medium senders emit clear signals; a Strong receiver who deviates by refusing all signals is worse off, because she forgoes profitable interactions with High senders. Both receiver types refrain from accepting silent senders provided that:

$$
p_{h}\left(1-r_{h}\right)<p_{l}
$$

If a Weak receiver switches to accepting only buried signals, she just loses profitable interactions with Medium senders. If (1)-(3) hold, the strategy profile is a strict Nash equilibrium, and therefore an evolutionary stable strategy (ESS) [9]. 
Here receivers can reliably distinguish between the three types of receivers thanks to costly signaling dynamics. According to costly signaling theory, signals can be reliable when the potential benefit of sending the signal, minus its cost, is higher for honest signalers than for liars [10]. In the most straightforward case, signal costs enforce honesty by causing complete separation between senders: all individuals with a given trait send the signal, while all individuals without the trait refrain from signaling (because they cannot afford to).

The standard burying equilibrium plays this trick twice. Separation between Low senders and other senders is achieved because signaling is prohibitively costly for Low types only. Separation between High and Medium types is slightly unconventional, yet still relies on costly signaling dynamics. By choosing to bury his signal, a sender pays an opportunity cost: he loses some profitable interactions with the Weak receivers who would have accepted a clear signal but fail to detect the buried signal. Because $r_{h}>r_{m}$, this opportunity cost is greater for a Medium signaler. When inequalities (1) and (2) above hold, this opportunity cost is enough to prevent Medium, but not High types, from burying.

We show in the appendix that the standard-burying strategy can invade a non-signaling strategy provided that conditions (1)(2) are met.

\section{Pooling burying equilibrium}

In the standard burying equilibrium, only High senders bury their signal, leaving Medium senders to openly brag about their positive traits. Here we show that the framework developed by Hoffman et al. is also able to account for norms of modesty that are upheld by everyone in the community: the signal-burying game has an equilibrium where every individual who sends a signal buries this signal.

To see why this is possible, consider the fact that receivers can take advantage of probabilistic information contained in a buried signal. Assume that the buried signals sent by High types are more likely to be detected than the ones sent by Medium types; then a significant proportion of the buried signals detected by receivers will come from High types. Under the right conditions, it may therefore be in the interest of Strong receivers to accept buried signals even in a population where both High and Medium types bury their signal. This happens when:

$$
p_{h} r_{h}>p_{m} r_{m}
$$

This is because, of all the senders a Strong receiver can be paired with, a proportion $p_{h}$ will be High types; of these, a proportion $r_{h}$ will see their buried signal detected by the receiver (left-hand side of the inequation). Similarly, a proportion $p_{m}$ will be Medium types; of these, a proportion $r_{m}$ will see their 
buried signal detected by the receiver (right-hand side). Therefore, of all senders a receiver can be paired with, a proportion $p_{h} r_{h}$ will be High types whose buried signal is detected, and a proportion $p_{m} r_{m}$ will be Medium types whose buried signal is detected. Because interacting with a Medium type is equally as bad as interacting with a High type is good, Strong receivers should accept buried signals when doing so guarantees them to meet, on average, more High than Medium senders, i.e. when (4) holds. ${ }^{2}$

Strong receivers reject silent senders when the absence of a signal is more likely to come from Medium or Low types than High types:

$$
p_{h}\left(1-r_{h}\right)<p_{m}\left(1-r_{m}\right)+p_{l}
$$

Because we assume that $p_{h}<p_{m}$, this condition is always met when (4) holds (because (4) and $p_{h}<p_{m}$ together imply that $r_{h}>r_{m}$ ).

Assume that Strong receivers only accept buried signals, and Weak receivers accept buried and clear signals. Depending on whether Weak receivers also have an incentive to accept silent senders, we have two variants of the pooling burying equilibrium. Weak receivers accept silent senders when the latter are more likely to be Medium or High types than Low types:

$$
p_{h}\left(1-r_{h}\right)+p_{m}\left(1-r_{m}\right)>p_{l}
$$

When this is the case, there is no opportunity cost to burying, so Medium and High types always bury their signal. Otherwise, when Weak receivers do not accept silent senders, High and Medium senders bury if

$$
\begin{aligned}
& r_{h}>q_{w} \\
& r_{m}>q_{w}
\end{aligned}
$$

In sum, when (6) holds, burying is Nash if (4) holds. When (6) does not hold, burying is Nash provided that (4)(7)(8) hold. In the first case, Weak receivers accept all senders, while in the second case Weak receivers only accept senders who send clear or buried signals. In both cases, Strong receivers only accept buried signals, while Medium and High senders send buried signals. We show in the appendix that the pooling burying strategy can invade a population of non-signalers provided that $(4)(7)(8)$ hold.

Note that the pooling burying strategy profile is not ESS: since no sender is sending clear signals, mutant Strong receivers who accept clear signals can invade the population via genetic drift. Nonetheless, the pooling equilibrium can be evolutionarily significant. We show this in two ways. First, we conduct individual-based simulations, which show that invasions by clear-signaling

\footnotetext{
${ }^{2}$ We also present a derivation of (4) in a Bayesian format in the appendix.
} 
strategies occur but are never long-lasting, such that, when the pooling burying strategy is Nash, the population spends most of the time at the pooling equilib-

rium. Second, we study a slightly modified version of the game where players can make mistakes, and show that the pooling strategy is ESS in this modified game.

\section{Individual-based simulations}

Analytical considerations show that the pooling burying strategy can be invaded, via neutral drift, by mutant Strong receivers who accept clear signals. When this happens, High and Medium senders are then better off sending a clear signal (since all receivers accept them). If $p_{h}<p_{m}$, accepting clear signals is detrimental to Strong receivers, so they switch to accepting only buried signals. Provided that the incentives of Medium and High senders favor burying, they go back to sending buried signals, and the population is at the pooling burying equilibrium again.

Therefore, when the conditions favoring the stability of the pooling burying strategy hold, analytical modeling predicts that the population will alternate between clear-signaling and burying strategies. In order to see whether one strategy would dominate this cycle, we performed individual-based computer simulations (see appendix for methods). Looking at populations in the last 1000 generations of each simulation, we find that for the simulations within the region of parameter space obeying $(4)(7)(8)$ and $p_{h}<p_{m}$, an average $87 \%$ of Medium senders, and $93 \%$ of High senders, send a buried signal (this region of parameter space corresponds to the blue triangle in Figure 1). Figure 2 presents a representative trajectory of the evolutionary dynamics. We see that although a typical population oscillates between clear-signaling and burying strategies, the population spends most of the time at the latter equilibrium. The result makes intuitive sense: in a population playing the clear-signaling strategy, Strong receivers who accept clear signals are strictly selected against, so the pooling-burying strategy rapidly invades. By contrast, in a population playing the pooling burying strategy, only neutral drift can increase the frequency of Strong receivers who accept clear signals, so such invasions are rare. 


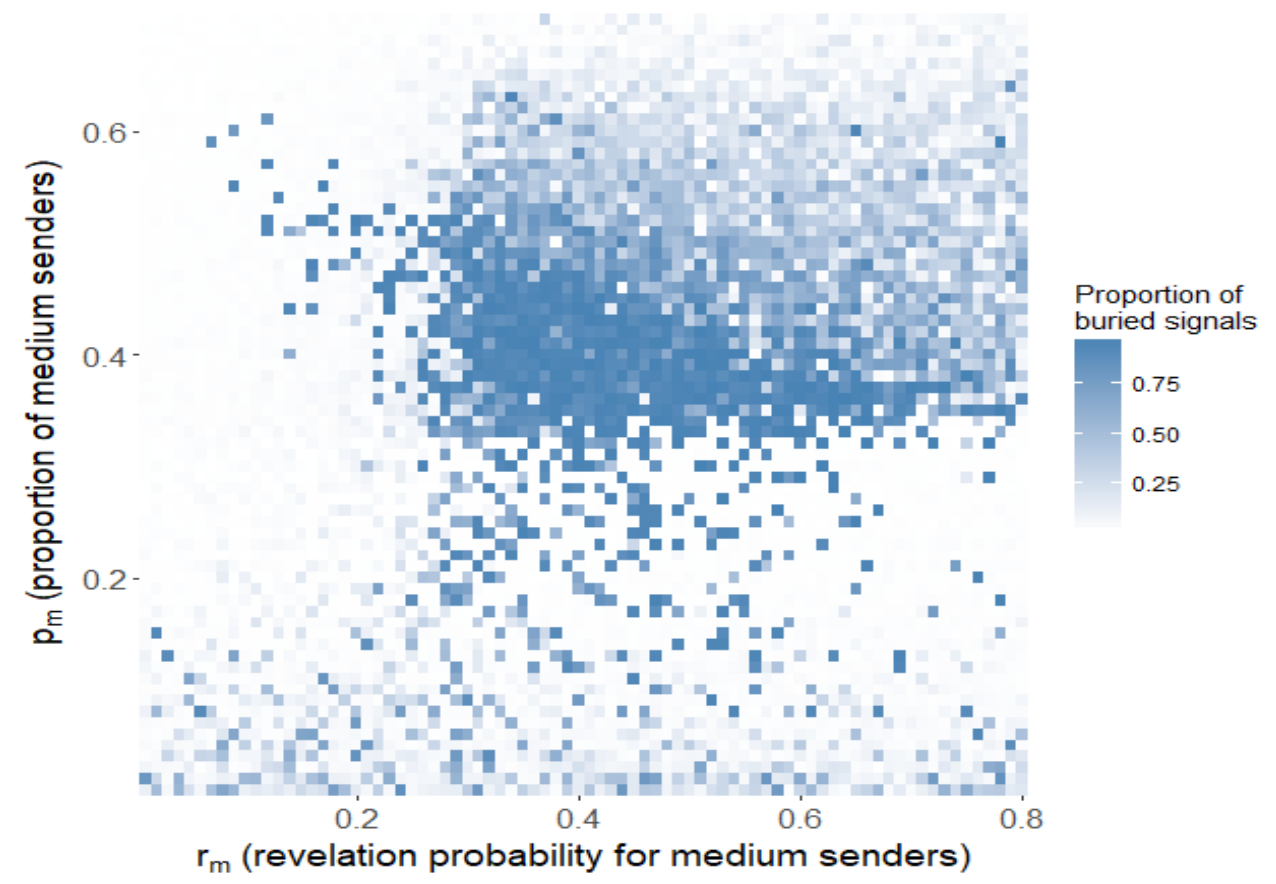

Figure 1: Average proportion of Medium senders sending buried signals in the last 1000 generations of each simulation, as a function of $p_{m}$ and $r_{m}$. Each pixel corresponds to one simulation. Other parameter values were fixed at $r_{h}=.8$, $r_{l}=.4, q_{w}=.3, q_{s}=.7, p_{l}=.3, p_{h}=1-p_{l}-p_{m}$. 


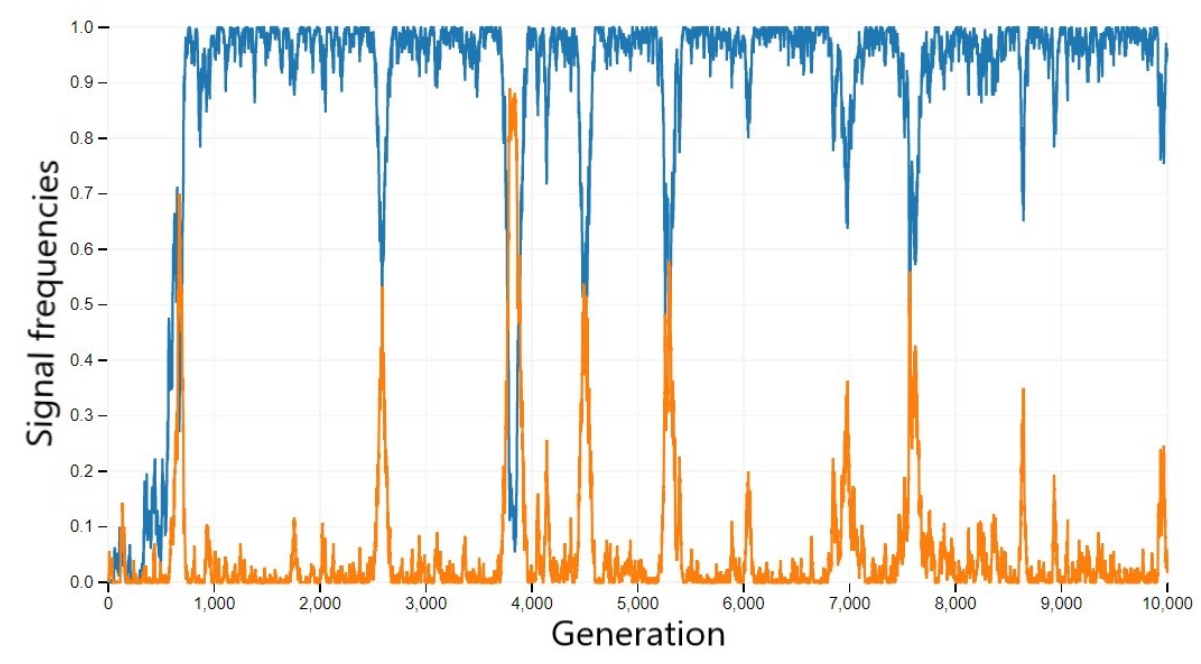

Figure 2: Frequency of Medium senders who send a clear (orange line) or buried signal (blue line) over time in a representative simulation run. Here, $r_{m}=.4$, and $p_{m}=.4$. Other parameter values were $r_{h}=.8, r_{l}=.4, q_{w}=.3, q_{s}=.7$, $p_{l}=.3, p_{h}=.3$.

\section{Analytical model with mistakes}

Strategies that are not evolutionarily stable can become so when the game is modified to take into account the possibility that individuals make mistakes, or that some individuals are unable to take a given action $[11,12]$. In the kinds of situations modeled by the signal-burying game, it is reasonable to assume that individuals who intend to bury their signal sometimes fail to do so. This is likely to occur, notably because individuals will try to steer a middle ground between making their signal too hard to detect (which would make them forgo too many interactions) and making it too obvious (in which case observers will stop considering the signal as 'buried' at all). Sometimes they will err on the side of making the signal detectable enough, and end up making the signal too obvious.

In the appendix, we formalize this argument by studying a game where the trade-off between detectability and discreetness is explicitly modeled. Here we simply analyze a game similar to the one described in the previous sections, with one modification: with probability $\epsilon$, a player who wishes to send a buried signal sends a clear signal instead. An alternative, but mathematically equivalent model would be to assume that $\epsilon$ represents a proportion of senders who are unable, for non-heritable reasons, to send buried signals.

In this game, the condition for Strong receivers to accept buried signals, under the pooling burying strategy, is the same as in the basic game: $p_{h} r_{h}>p_{m} r_{m}$ 
(inequation(4)). Since Medium and High types have the same error rate, introducing mistakes does not change the diagnosticity of buried signals.

Strong receivers reject silent senders provided that

$$
p_{h}\left(1-r_{h}\right)<p_{m}\left(1-r_{m}\right)+\frac{p_{l}}{1-\epsilon}
$$

Just as in the no-mistakes model, this condition is always met provided that (4) and $p_{h}<p_{m}$ are true.

Weak receivers reject silent senders if:

$$
p_{h}\left(1-r_{h}\right)+p_{m}\left(1-r_{m}\right)<\frac{p_{l}}{1-\epsilon}
$$

When (9) does not hold, High and Medium senders always bury. Otherwise, they have an incentive to bury provided that:

$$
\begin{aligned}
& (1-\epsilon) r_{h}+\epsilon q_{w}>q_{w} \\
& (1-\epsilon) r_{m}+\epsilon q_{w}>q_{w}
\end{aligned}
$$

These inequalities reduce to $r_{h}>q_{w}$ and $r_{m}>q_{w}$, which are simply inequalities (7) (8).

Medium and High types are equally likely to send a clear signal by mistake; therefore, because $p_{h}<p_{m}$, a Strong receiver should not accept clear signals, and the clear-signaling strategy cannot invade by neutral drift. Therefore, if the pooling burying strategy is a Nash equilibrium, it is also ESS.

\section{Discussion}

We have shown that, in addition to the burying equilibrium identified by Hoffman et al., the signal-burying game has a "pooling burying equilibrium" where all individuals who send a signal bury that signal. These two different burying equilibria rely on very different dynamics.

The pooling burying strategy is stable because Strong receivers make optimal use of the probabilistic information contained in the signal. When buried signals sent by High types are more likely to be detected than those sent by Medium types, most detected signals come from High senders (or at least, the proportion of detected signals that come from High senders is greater than the ratio of High to Medium senders in the population), which makes burying status a cue to sender quality. 
As a result, both Medium and High senders have an incentive to bury their signal in order to attract Strong receivers. By doing so, they trade off the conspicuousness of their signal against its inferential content: they are willing to reduce the number of receivers they reach, in order to present themselves in the best light. One may describe senders who play this strategy as imposing a Handicap on themselves. Deliberately diminishing the visibility of their signal hurts their chances of interacting with Weak receivers, who are just looking for evidence that they are not Low types. Furthermore, the magnitude of this handicap must be larger for Medium than for High types $\left(r_{m}<r_{h}\right)$ for the strategy to be stable. Therefore, the situation bears a superficial similarity to the Handicap principle theorized by Zahavi [4] and later formalized by Grafen [13], whereby costly signals can promote communication when interests conflict.

However, standard costly signaling models rely on a payoff differential between sender types; namely, the marginal payoff of sending the signal must be higher for honest senders than dishonest individuals [10]. This payoff differential makes the signal reliable, because the individuals who can afford to send the signal are disproportionately likely to be honest signalers. By contrast, when playing the pooling burying strategy, Medium senders earn lower payoffs than High senders, but this payoff differential is immaterial to the inferential value of burying. Indeed, in a model where Medium types earn interaction payoffs ten times greater than High types, burying would give Medium senders a higher marginal payoff than High types, yet Strong receivers would still use buried signals as a cue of sender quality.

The standard burying strategy does rely on costly signaling: burying is a reliable cue to High type because the opportunity cost of burying dissuades Medium, but not High senders from burying. It is a separating equilibrium, where only High senders send a buried signal. By contrast, the existence of the pooling burying equilibrium shows that modesty can make a signal more convincing even in the absence of a separation between sender types.

\section{Conclusion}

Game theorists are starting to better understand why people strategically encrypt some of the signals they send. People use indirect speech to maintain plausible deniability when making a bribe, threat, or sexual advance, or to prevent sensitive information from becoming common knowledge [14]. They also communicate using coded messages that can only be understood by people with the right pre-existing knowledge, in order to reach potential allies without antagonizing individuals who would dislike the message [15]. The signal-burying game highlights another mechanism: making a signal harder to notice can itself act as a signal. If senders with the most desirable qualities are also the most adept at conveying these qualities in a subtle fashion, then subtlety can reliably 
convey their possession. The present paper offers the additional insight that even the senders who are bad at skillfully obfuscating their message may find it worthwhile to do so. Doing otherwise would be an implicit confession that they are not skillful buriers, and therefore not high-quality individuals. In many cases maintaining ambiguity about one's quality is worth the risk of not getting the message through.

\section{References}

[1] De Freitas J, DeScioli P, Thomas KA, Pinker S. Maimonides Ladder: States of Mutual Knowledge and the Perception of Charitability. Journal of experimental psychology General. 2018;

[2] Trivers RL. The evolution of reciprocal altruism. The Quarterly review of biology. 1971;46(1):35-57.

[3] Spence M. Job Market Signaling. The Quarterly Journal of Economics. 1973;87(3):355-374.

[4] Zahavi A. Mate selection - a selection for a handicap. Journal of theoretical Biology. 1975;53(1):205-214.

[5] Miller G. The mating mind: How sexual choice shaped the evolution of human nature. Anchor; 2000.

[6] Barclay P. Strategies for cooperation in biological markets, especially for humans. Evolution and Human Behavior. 2013;34(3):164-175.

[7] Sznycer D, Al-Shawaf L, Bereby-Meyer Y, Curry OS, De Smet D, Ermer E, et al. Cross-cultural regularities in the cognitive architecture of pride. Proceedings of the National Academy of Sciences. 2017;114(8):1874-1879.

[8] Hoffman M, Hilbe C, Nowak MA. The signal-burying game can explain why we obscure positive traits and good deeds. Nature Human Behaviour. 2018;p. 1.

[9] Maynard Smith J. Evolution and the Theory of Games. Cambridge university press; 1982 .

[10] Higham JP. How does honest costly signaling work? Behavioral Ecology. 2013;25(1):8-11.

[11] Boyd R. Mistakes allow evolutionary stability in the repeated prisoner's dilemma game. Journal of theoretical Biology. 1989;136(1):47-56.

[12] Sherratt TN, Roberts G. The importance of phenotypic defectors in stabilizing reciprocal altruism. Behavioral Ecology. 2001;12(3):313-317.

[13] Grafen A. Biological signals as handicaps. Journal of theoretical biology. 1990;144(4):517-546. 
[14] Pinker S, Nowak MA, Lee JJ. The logic of indirect speech. Proceedings of the National Academy of sciences. 2008;105(3):833-838.

[15] Smaldino PE, Flamson TJ, McElreath R. The evolution of covert signaling. Scientific reports. 2018;8(1):4905.

\section{Appendix}

\section{Derivation of inequality (4) using Bayes' rule}

Let us assume that Medium and High senders all send a buried signal. A receiver who detects a buried signal can infer the probability that the sender is a High type using Bayes' rule:

$$
P(\text { High } \mid \text { buried })=\frac{P(\text { buried } \mid \text { high }) P(\text { high })}{P(\text { buried })}
$$

Where $P($ buried $\mid$ high $)$ is the probability that a sender sends a buried signal that gets detected, given that the sender is a High type. Since by assumption all High senders send a buried signal, this is equal to $r_{h}$. Therefore we have:

$$
P(\text { High } \mid \text { buried })=\frac{r_{h} p_{h}}{r_{h} p_{h}+r_{m} p_{m}}
$$

A similar argument reveals that:

$$
P(\text { Medium } \mid \text { buried })=\frac{r_{m} p_{m}}{r_{h} p_{h}+r_{m} p_{m}}
$$

A Strong receiver should accept a buried signal when $P($ High $\mid$ buried $)>$ $P($ Medium $\mid$ buried $)$, that is, when $r_{h} p_{h}>r_{m} p_{m}$

\section{Invasion potential of burying strategies}

Here we explore the conditions under which a burying strategy can invade a non-signaling strategy. A non-signaling strategy is a strategy where no sender ever signals, and receivers are predisposed to always reject senders, no matter which signal they receive.

Assume every agent initially plays the non-signaling strategy. Since no signals are sent, via genetic drift receivers eventually start accepting all signals. Then, Medium and High senders start sending clear signals. Since $p_{h}<p_{m}$, Strong receivers are harmed by clear signalers and stop accepting clear signals.

If $r_{h}>q_{w}$, and $r_{m}<q_{w}$, there is now an incentive for High senders (but not Medium senders) to send buried signals, and the standard burying strategy invades. That is, the strategy can invade if $r_{h}>q_{w}$, and $r_{m}<q_{w}$ (conditions (1)(2)). 
If, instead, $r_{h}>q_{w}$ and $r_{m}>q_{w}$, there is now an incentive for High senders and Medium senders to send buried signals. Strong receivers keep accepting these buried signals if $p_{h} r_{h}>p_{m} r_{m}$, and the pooling burying strategy invades. That is, the strategy can invade if $p_{h} r_{h}>p_{m} r_{m}, r_{h}>q_{w}$ and $r_{m}>q_{w}$ (conditions $(4)(7)(8))$.

\section{Individual-based simulations}

Each simulation involved a population of 300 senders, and a population of 300 receivers, allowed to evolve for 10000 generations. For each simulation, we recorded the average frequency of each allele during the last 1000 generations. At the beginning of each generation, each sender was paired randomly with a receiver. Then each pair played a signal-burying game, after which all agents died and reproduced asexually. Within each population, an agent's expected number of offspring was equal to the ratio of its total payoff to the average payoff in that population (payoffs were first standardized such that the agent with the lowest payoff had payoff 1 ; this was achieved by subtracting, to the payoff of each agent, the payoff of the agent with the lowest payoff, then adding 1 to the payoff of each agent. This procedure ensured that no agent had a negative payoff before the selection phase). The size of each population was kept constant at 300 agents during a simulation.

Sender and receiver type were non-heritable, and determined at random, according to the probabilities $p_{h}, p_{m}, p_{l}, q_{s}$ and $q_{w}$, for each agent at the start of every generation. A sender's genotype consisted in 3 genes, determining the sender's behavior for each possible type it could be (High, Low, or Medium); each gene could take either Bury, Clear or Quiet as alternative alleles. A receiver's genotype consisted in 6 genes, determining the receiver's behavior (Accept or Reject) for each combination of receiver's type (Strong or Weak) and sender's behavior (buried signal, clear signal, no signal). Each agent inherited its parent's genotype, subject to mutation: with independent probability .002 , each gene would take a new, randomly determined value (note that this process was allowed to generate a "new" value identical to the pre-mutation value).

We conducted 5600 simulations, while varying the values of $r_{m}$ (from .01 to .8) and $p_{m}$ (from .01 to .7) in .01 increments. Other parameter values were fixed at $r_{h}=.8, r_{l}=.4, q_{w}=.3, q_{s}=.7, p_{l}=.3, p_{h}=1-p_{l}-p_{m}$. Low senders incurred a cost of 10 when sending a signal. Each simulation was initialized with a population where senders never signaled and receivers were predisposed to reject all senders, regardless of the signal they would receive. Simulation software is written in JavaScript; script and data for the simulations are available in the electronic supplementary materials. 


\section{Modeling the trade-off between detectability and discreet- nesss}

We study a signal-burying game identical to the basic version analyzed in the main text, except that the probability of detection of a buried signal $(r)$ is determined endogenously. Instead of making a discrete decision to bury or not, senders can decide how much to bury their signal, via a continuous variable $b \in[0,1]$. A sender's signal is detected by the receiver with probability $r=1-\alpha b$. Crucially, a receiver who detects a signal does not always notice that it was buried. The deeper a sender buries the signal, the more likely it is to be identified as buried, provided it is detected: this happens with probability $b$. Therefore senders are faced with a trade-off: the more they bury a signal, the less likely it is to be detected, but the more likely it is to be identified as having been buried. We allow $\alpha$ to differ between High and Medium senders. $\alpha_{h}<\alpha_{m}$, for instance, would mean that High senders are smarter than Medium senders about how they hide their signal. We assume that $p_{h}\left(1-r_{h}\right)+p_{m}\left(1-r_{m}\right)<p_{l}$, such that receivers do not accept silent senders.

Assume that Strong receivers accept only buried signals, while Weak receivers accept all signals. Then, the expected payoff of a High sender is given by:

$$
E(h)=r_{h}\left(b_{h} q_{s}+q_{w}\right)
$$

because a proportion $r_{h}$ of receivers detect the signal; of these, all Weak receivers accept the signal, while only the Strong receivers who identify the signal as buried accept it. This is equivalent to:

$$
E(h)=\left(1-\alpha_{h} b_{h}\right)\left(b_{h} q_{s}+q_{w}\right)
$$

The derivative of $E(h)$ with respect to $b_{h}$ is

$$
E(h)^{\prime}=-2 \alpha_{h} q_{s} b_{h}-\alpha_{h} q_{w}+q_{s}
$$

To find the value of $b_{h}$ that maximizes $E(h)$, we set $E(h)^{\prime}=0$ and solve for $b_{h}$. This yields:

$$
b_{h}=\frac{q_{s}-\alpha_{h} q_{w}}{2 \alpha_{h} q_{s}}
$$

If the right-hand side of the above equation is below 0 or above 1 , then $b_{h}=0$ or $b_{h}=1$, respectively (since $b_{h}$ is constrained to range between 0 and 1 ).

Similarly, $E(m)$ is maximized when

$$
b_{m}=\frac{q_{s}-\alpha_{m} q_{w}}{2 \alpha_{m} q_{s}}
$$

with the same constraint as above on the range of $b_{m}$. 
When are Strong receivers better off accepting buried signals? Of all the senders they encounter, a proportion $p_{h}$ are High types; of these, a proportion $r_{h}$ see their signal detected; of these, a proportion $b_{h}$ are identified as being buried. Therefore, of all senders a receiver can be paired with, a proportion $p_{h} r_{h} b_{h}$ will be High types whose signal is detected and identified as buried; similarly, a proportion $p_{m} r_{m} b_{m}$ will be Medium types whose signal is detected and identified as buried. Strong receivers are therefore better off accepting buried signals when

$$
p_{h} r_{h} b_{h}>p_{m} r_{m} b_{m}
$$

where $r_{h}=1-\alpha_{h} b_{h}$ and $r_{m}=1-\alpha_{m} b_{m}$

For the burying equilibrium to be stable, it must also be the case that Strong receivers are better off rejecting clear signals:

$$
p_{h} r_{h}\left(1-b_{h}\right)<p_{m} r_{m}\left(1-b_{m}\right)
$$

A final condition for burying to be a Nash equilibrium is that High senders bury their signal to some extent, that is $b_{h}>0$. From (12), we see that this happens when:

$$
\alpha_{h}<\frac{q_{s}}{q_{w}}
$$

When (14)-(16) hold, the burying strategy is Nash. In order for it to be ESS, we also need some signals to be identified as clear at least a fraction of the time; otherwise selection on receivers' reaction to clear signals disappears. This condition is met when $b_{m}<1$; from (13), this is equivalent to $\alpha_{m}>\frac{q_{s}}{2 q_{s}+q_{w}}$.

Note that this burying equilibrium encompasses as special cases the standard burying equilibrium (with High types burying and Medium types always sending clear signals) when $\alpha_{h}<\frac{q_{s}}{q_{w}}$ and $\alpha_{m}>\frac{q_{s}}{q_{w}}$, and the pooling equilibrium (with every agent who sends a signal burying that signal to some extent) when both $\alpha_{m}$ and $\alpha_{h}$ are smaller than $\frac{q_{s}}{q_{w}}$.

As a numerical example, when $q_{s}=.7, q_{w}=.3, p_{h}=.3, p_{m}=.4, \alpha_{h}=.5$ and $\alpha_{m}=.8$, we have:

$$
\begin{aligned}
& b_{h}=.79, r_{h}=.61 \\
& b_{m}=.41, r_{m}=.67
\end{aligned}
$$

Then $p_{h} r_{h} b_{h}=.14, p_{m} r_{m} b_{m}=.11, p_{h} r_{h}\left(1-b_{h}\right)=.04, p_{m} r_{m}\left(1-b_{m}\right)=.16$, thus inequalities (14) and (15) hold, and the burying strategy is ESS. Note that here, unlike the models in the main text, signals from High types happen to be slightly less likely to be detected than signals from Medium types. Buried signals still function as a cue to High quality because signals from High types are less "obvious": they are more likely to be identified as having been buried 
than signals from Medium types. Here, burying evolves because High types are better at making apparent the encrypted nature of their message. 